\title{
Resistive Water Sensors Based on PEDOT: PSS-g- PEGME copolymer and Laser Treatment for Water Ingress Monitoring Systems
}

Seongin Hong1, ${ }^{1}$, Jung Joon Lee ${ }^{1, \dagger}$, Srinivas Gandla1, Junwoo Park', Haewon Cho ${ }^{1}$, and Sunkook Kim ${ }^{1, *}$

1 School of Advanced Materials Science \& Engineering, Sungkyunkwan University, Suwon, 440-745, Republic of Korea; seonkuk@skku.edu.

Keywords: resistive water sensor, water ingress monitoring systems, PEDOT: PSS, water resistance, laser treatment 


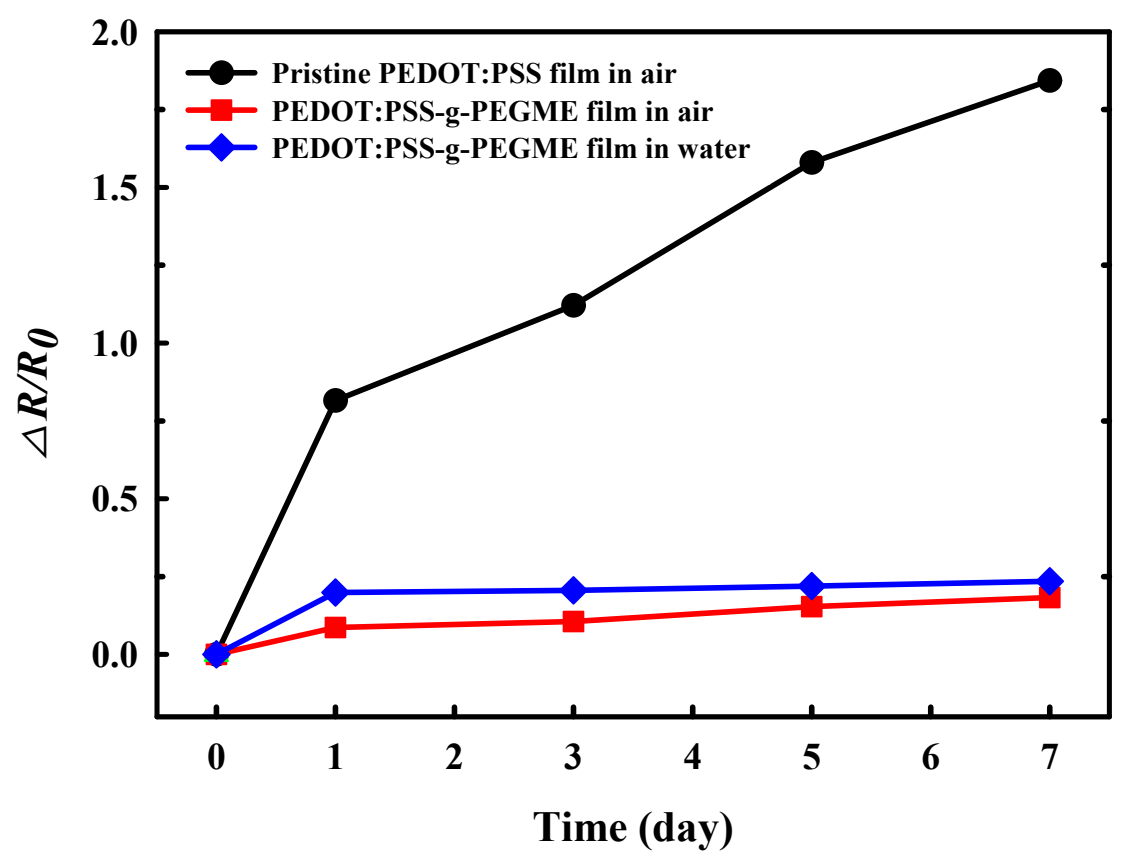

Figure S1. The relative resistance changes of pristine PEDOT:PSS and PEDOT:PSS-gPEGME copolymer film over time exposed to air or water.

Table S1. Statistical data of result extracted from Figure 5a.

\begin{tabular}{|c|c|c|c|c|c|c|}
\hline \multicolumn{2}{|c|}{} & \multicolumn{5}{|c|}{ Water level (cm) } \\
\cline { 3 - 7 } \multicolumn{2}{|c|}{} & 0 & 1 & 2 & 3 & 4 \\
\hline \multirow{3}{*}{$\begin{array}{c}\text { Current } \\
(\mu \mathrm{A})\end{array}$} & Average & 0.13 & 0.71 & 1.26 & 1.94 & 2.74 \\
\cline { 2 - 7 } & $\begin{array}{c}\text { Standard } \\
\text { deviation }\end{array}$ & 0.02 & 0.08 & 0.04 & 0.02 & 0.03 \\
\hline
\end{tabular}

